\title{
Migrating Birds Optimization for Flow Shop Sequencing Problem
}

\author{
Vahit Tongur ${ }^{1}$, Erkan Ülker² \\ ${ }^{1}$ Computer Engineering Department, Necmettin Erbakan University, Konya, Turkey \\ ${ }^{2}$ Computer Engineering Department, Selçuk University, Konya, Turkey \\ Email: vtongur@konya.edu.tr, eulker@selcuk.edu.tr
}

Received November 2013

\begin{abstract}
FSSP is a typical NP-Hard problem which is desired to be minimum makespan. This study considers Migrating Birds Optimization (MBO) which is metaheuristic approach for the solution of Flow Shop Sequencing Problem (FSSP). As the basic MBO algorithm is designed for discrete problems. The performance of basic MBO algorithm is tested via some FSSP data sets exist in literature. Obtained results are compared with optimal results of related data sets.
\end{abstract}

Keywords

Migrating Birds Optimization; Flow Shop Sequencing Problem; Metaheuristic Optimization

\section{Introduction}

Flow shop sequencing problem (FSSP) is a production planning problem. Production planning problem in today's manufacturing systems planning process has a very important place. Therefore a lot of research has been done on scheduling problems. Researchers have developed various heuristic approaches because of most of the scheduling problems in NP-hard class.

There are close optimum solutions for large scaled integrated optimization problems as an optimum solution. Close optimum solutions are generally found via heuristic algorithms. While some heuristic algorithms start to solution from zero and represent a graded solution, some of them start from a complete solution and try to enhance the existing solution [1,2]. Most of the metaheuristic algorithms can also be called as neighboring or local search procedures. Metaheuristic algorithms are a common form of enchanging algorithms which find a better solution by looking for neighboring of existing solution in each iteration [2,3]. Neighboring attitude of each metaheuristic algorithm is different. It can be said that differences of these algorithms, which basically have the same targets, are sourced from their neighboring attitudes. Until now, some metaheuristic algorithms such as genetic algorithm [4,5], tabu search [6,7], ant colony algorithm [8] are solved by FSSP problems.

Flow shop sequencing problem minimizing the time between the beginning of perform of the first job on the first machine and the completion of perform of the last job on the last machine. This time is called make span. Assumptions for this problem are as follows.

- Every job has to be processed at maximum once on machine. 
- Every machine processes only one job at a time.

- Every job is processed at maximum on one machine at a time.

- The preparation times of the operations are included in the processing time and do not depend on the sequence.

- The operating sequences of the jobs are the same on every machine [9].

FSSP can be formulated as below;

For $n$ jobs and $m$ machine,

Job permutation $\pi=\left\{\pi_{1}, \pi_{2}, \pi_{3}, \cdots \pi_{n}\right\}$ and $p(i, j)$ processing time for job $i$ on machine $j$.

$$
\begin{aligned}
& C\left(\pi_{1}, 1\right)=p\left(\pi_{1}, 1\right) \\
& C\left(\pi_{i}, 1\right)=C\left(\pi_{i-1}, 1\right)+p\left(\pi_{i}, 1\right) \text { for } i=2, \cdots, n \\
& C\left(\pi_{1}, j\right)=C\left(\pi_{1}, j-1\right)+p\left(\pi_{1}, j\right) \text { for } j=2, \cdots, m \\
& C\left(\pi_{i}, j\right)=\max \left\{C\left(\pi_{i-1}, j\right), C\left(\pi_{i}, j-1\right)\right\}+p\left(\pi_{i}, j\right) \\
& \text { for } i=2, \cdots n \text {; for } j=2, \cdots, m
\end{aligned}
$$

Makespan $C_{\max }=C\left(\pi_{n}, m\right) \quad[4,5]$.

Organization of the article is follows; In the second section, MBO algorithm is mentioned. After telling application of FSSP problem to MBO algorithm in third section, numeric results of MBO algorithm, which is applied to FSSP problem, is given in fourth section. In the final section, obtained results of the study are assessed.

\section{Migrating Birds Optimization Algorithm}

Migrating Birds Optimization algorithm (MBO) is revealed by Duman et al. [2]. They are inspired from the flight position of migratory birds while they migrate as a flock.

Migrating Birds Optimization algorithm (MBO) is revealed as a result of studies on V formation of the birds, which provides energy saving in flying long distances, during the migration. The reason of $\mathrm{V}$ formation in actual life is commented as minimizing the necessary energy of flight by using air turbulence of leader bird's flapping. It is thought that birds fly in a specific angle and distance with the leader bird for using this energy saving. Wing distances of different migrating birds, which fly in this formation, is different. As wing distances can change the stress and position of the turbulence, the size of formation can be different for different birds (distance and angle values between leader bird and the others) [2].

Leader bird in the flock is the one which spends the most energy. The other birds after it, take the advantage of leader bird. When the leader bird gets tired, another bird in the flock takes its position and the tired bird goes back side of the flock.

MBO is a neighbouring research technique including algorithm. Each bird in V formation represents a solution. Starts with first solution (leader bird) and goes on till tail. Each solution has solutions which try to advance it. These neighboring solutions provide the opportunity of local searches in the position of existing solution. If the neighbor solutions are better than the existing one, they are replaced. Apart from the other metaheuristic algorithms, there is another efficiency mechanism in MBO algorithm. This mechanism transfers the best unused neighbor solutions to following solution. In algorithm, this situation is called as sharing the neighbors. In this way, existing solution enhances itself by using not only its own produced solutions but also neighbor solutions which come from other solutions. This neighbor sharing is concluded with sharing of the solution by all birds (except for the last two birds in the tail). After that the leader bird is changed and new neighborhoods and neighbor sharing, which will be produced by new leader bird, is restarted. This process continues until determined iteration value is reached [2].

Pseudo code of MBO algorithm is given below; 


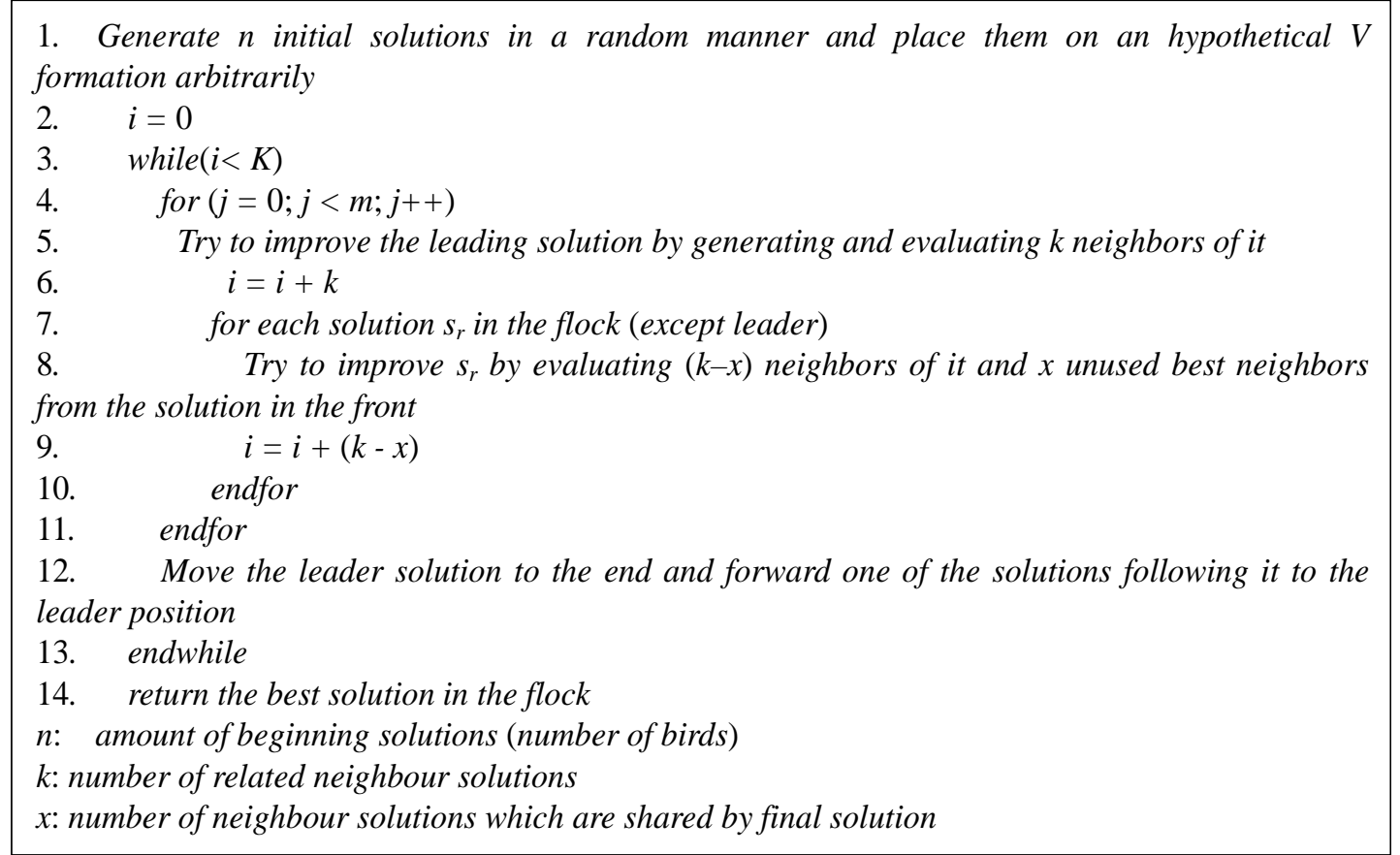

$n$ : amount of beginning solutions (number of birds)

$k$ : number of related neighbour solutions

$x$ : number of neighbour solutions which are shared by final

$m$ : number of lap

$K$ : iteration limit [2]

Algorithm shows similarities with real life of migrating birds. $n$ value shows number of birds. First birds are in $\mathrm{V}$ formation. An existing solution is produced for each bird. $k$ value is thought as speed of a bird in real life. The bird should fly slowly for a more detailed discovery around itself. So $k$ parameter is expected to have low values in order to be able to reach better solutions. $x$ value is thought as wing distance of the bird (WTS length in Figure 1). In experimental studies of Lissaman and Schollenberger, optimum wing distance for the 1.5 meter wing length birds is determined as $16 \mathrm{~cm}[2,10]$. Which means that $x$ parameter will be appointed very low values. $m$ value is thought as number of flutter of the bird. It is the flutter number until leader bird gets tired and goes back for resting. According to algorithm, when leader bird gets tired it goes to the back of flock and its following bird becomes leader bird. As the birds are in V formation, first the bird on the left of the leader bird takes the position of leader bird. In this condition leader bird goes to the back line of the left order. In the next change, the bird on the right of the leader bird takes its position and leader goes to the right back of the flock. And the iteration number of algorithm should be as much as that each bird would become leader at least once [2].

\section{Solution of FSSP with MBO Algorithm}

Basic MBO algorithm is tested on quadratic assignment problems (QAP) [11]. Its structure resembles to genetic algorithm partially. While solving QAP problems, it is incorporated into problem permutation line as in genetic algorithm. While determining neighbor solutions, only one pair of existing solution is replaced. So, producing neighborhood is thought to enable a more detailed local search.

MBO algorithm also has a similar structure of chromosome which is used in genetic algorithm. As discrete problems such as QAP are designed, basic MBO algorithm is kept the same for FSSP. Also enhanced parameters are used which are obtained via QAP samples.

As in most metaheuristic algorithms, MBO starts with a beginning solution. In the beginning, permutation of all jobs on all machines is created for each bird and make span is determined. For $\mathrm{n}$ job and $\mathrm{m}$ machine $m \times n$ matrix is formed. Therefore all jobs and all machines are placed in the $m \times n$ matrix. After that neighboring is produced for each bird in the flock in enough amounts and if the best neighbor of the existing solution is better 
than the existing solution, neighbor solution is replaced by existing solution. Neighbors are produced faithfully to original algorithm. The best unused solution is transferred to the bird in the back order. And the worst unused solution of the related bird's back order is dismissed.

\section{Experimental Results}

In the application, MBO algorithm is tested Taillard's flow shop sequence problems [12]. All tested problems are executed 10 times by keeping the starting point same and results are averaged. Iteration value of basic MBO algorithm is depended upon the produced neighbor amount and size of the problem. This value determined as $n^{4}$ ( $n=$ job count). In both problems, static parameter values are given in Table $\mathbf{1}$.

Values for 10 times execution of all tested problems are saved. According to these values, maximum, minimum and average values of makespan are given in Tables 2-4 which are calculated by considering average of 10 studies for some iteration.

Best known upper bounds of tested Taillard's instances are given in Table 5.

\section{Conclusion}

In this article, solutions are seeked for the well known optimization problem flow shop sequencing problem with migrating birds optimization (MBO) algorithm which is a new metaheuristic approach. Developed algorithm is experimentally tested by well known data set which are chosen from Taillard's benchmark and compared

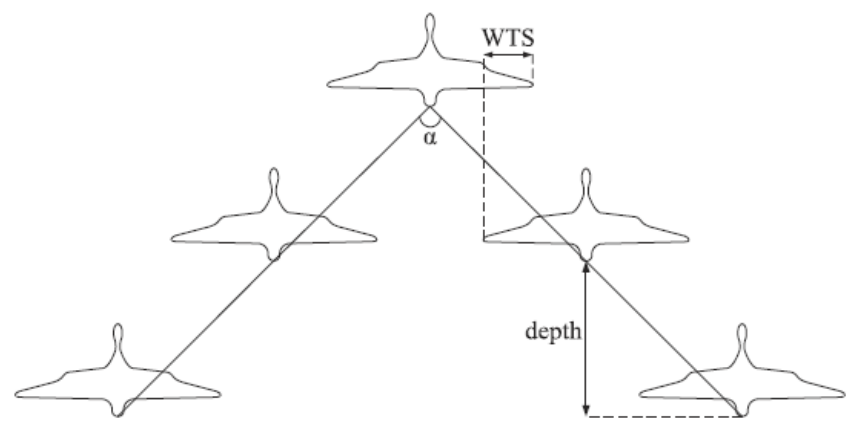

Figure 1. The V formation.

Table 1. Starting parameter values.

\begin{tabular}{ccccc}
\hline & \multicolumn{3}{c}{ MBO Parameters } \\
\cline { 2 - 4 } & $k$ & $x$ & $m$ \\
\hline 51 & 3 & 1 & 10 \\
\hline
\end{tabular}

Table 2. Maximum, minimum and average values for $20 \times 5$ instance1.

\begin{tabular}{cccc}
\hline Leader Replace Number & \multicolumn{2}{c}{$20 \times 5$ instance1 } \\
\cline { 2 - 4 } & \multicolumn{1}{c}{ Min. } & Max. & Avg. \\
\hline 5 & 1334.4 & 1426 & 1374.5 \\
10 & 1297 & 1340.5 & 1311.6 \\
50 & 1295.7 & 1305.9 & 1297.9 \\
100 & 1291.2 & 1297 & 1295.4 \\
156 & 1288.1 & 1296.8 & 1291.3 \\
\hline
\end{tabular}


Table 3. Maximum, minimum and average values for $20 \times 10$ instance1.

\begin{tabular}{cccc}
\hline Leader Replace Number & \multicolumn{2}{c}{$20 \times 10$ instance 1} \\
& Min. & Max. & Avg. \\
1 & 1755.6 & 1890.4 & 1825 \\
10 & 1658.8 & 1758.3 & 1711.1 \\
50 & 1639.8 & 1712.6 & 1676.3 \\
100 & 1601.9 & 1646.3 & 1624.7 \\
156 & 1594.2 & 1635 & 1615.3 \\
\hline
\end{tabular}

Table 4. Maximum, minimum and average values for $20 \times 20$ instance1.

\begin{tabular}{cccc}
\hline \multirow{2}{*}{ Leader Replace Number } & \multicolumn{2}{c}{$20 \times 20$ instance1 } \\
\cline { 2 - 4 } & Min. & Max. & Avg. \\
\hline 5 & 2474.9 & 2629.6 & 2552.4 \\
10 & 2395.4 & 2497.7 & 2448.7 \\
50 & 2364.2 & 2455.1 & 2413.5 \\
100 & 2325.6 & 2392.6 & 2355.4 \\
156 & 2320.6 & 2375.3 & 2343 \\
& 2318.9 & 2362.9 & 2337.7 \\
\hline
\end{tabular}

Table 5. Upper bound values of tested problems.

\begin{tabular}{ccc} 
& \multicolumn{3}{c}{ Upper Bound Values } \\
\hline $20 \times 5$ ins.1 & $20 \times 10$ ins.1 & $20 \times 20$ ins.1 \\
\hline 1278 & 1582 & 2297 \\
\hline
\end{tabular}

according to optimal solutions. As a result of proposed study, forFSSP; it is seen that MBO algorithm follows a performance progress and has a more consistent approach to result.

\section{Acknowledgements}

This study has been supported by Scientific Research Project of Necmettin Erbakan University.

\section{References}

[1] Glover, F. and Kochenberger, G.A. (2003) Handbook of Metaheuristics. Kluwer Academic Publishers.

[2] Duman, E., Uysal, M. and Alkaya, A.F. (2012) Migrating Birds Optimization: A New Metaheuristic Approach and Its Performance on Quadratic Assignment Problem. Information Sciences, 217, 65-77. http://dx.doi.org/10.1016/j.ins.2012.06.032

[3] Ahuja, R.K., Ergun, O., Orlin, J.B. and Punnen, A.P. (2002) A Survey of Very Large Scale Neighborhood Search Techniques. Discrete Applied Mathematics, 123, 75-102. http://dx.doi.org/10.1016/j.ins.2012.06.032

[4] Reeves, C.R. (1995) A Genetic Algorithm for Flowshop Sequencing. Computers \& Operations Research, 22, 5-13. http://dx.doi.org/10.1016/0305-0548(93)E0014-K

[5] Reeves, C.R. and Yamada, T. (1998) Genetic Algorithms, Path Relinking and the Flowshop Sequencing Problem. Evolutionary Computation Journal (MIT Press), 6, 230-234. 
[6] Armentano, V.A. and Ronconi, D.P. (1999) Tabu Search for Total Tardiness Minimization in Flowshop Scheduling Problems. Computers \& Operations Research, 26, 219-235.

[7] Ekşioğlu, B., Ekşioğlu, S.D. and Jain, P. (1998) A Tabu Search Algorithm for the Flowshop Scheduling Problem with Changing Neighborhoods. Computer \& Industrial Engineering, 1-11.

[8] Rajendran, C. and Ziegler, H. (2004) Ant-Colony Algorithms for Permutation Flowshop Scheduling to Minimize Makespan/Total Flowtime of Jobs. European Journal of Operational Research, 155, 426-438.

[9] Taillard, E. (1989) Some Efficient Heuristic Methods for the Flow Shop Sequencing Problem.

[10] Lissaman, P.B.S. and Shollenberger, C.A. (1970) Formation Flight of Birds. Science, 168, 1003-1005. http://dx.doi.org/10.1126/science.168.3934.1003

[11] Burkard, R.E., Çela, E., Karisch, S.E. and Rendl, F. (2010) QAPLIB Aquadratic Assignment Problem Library. http://www.opt.math.tu-graz.ac.at/qaplib/

[12] Taillard, E. (2013) Scheduling Instances. http://mistic.heig-vd.ch/taillard/problemes.dir/ordonnancement.dir/ordonnancement.html 